\title{
A HYBRID MODEL FOR EVACUATION SIMULATION AND EFFICIENCY OPTIMIZATION IN LARGE COMPLEX BUILDINGS
}

\author{
Hao Yuan , Guo Yu ${ }^{2}$, Yifan Ma , Jieneng $\mathrm{Chen}^{3}$, Xiongda Chen ${ }^{2}$ \\ School of Software Engineering, Tongji University, China \\ ${ }^{3}$ School of Electronics and Information Engineering, Tongji University, China
}

\begin{abstract}
The Cellular Automaton(CA) and Artificial Potential Field(APF) method, as well as other theories, are traditional to simulate the flow of people.A refine model of CA, combined with adapted Ant Colony model, as well as the APF is delivered to simulate the evacuation process in large buildings. An estimation of the total evacuation time within one floor in the Louvre is obtained by applying this model. The bottlenecks are identified alongside the evacuation routes. The applicability and flexibility of this model are proved.
\end{abstract}

\section{KEYWORDS}

Evacuation Model, Cellular Automaton, Artificial Potential Field, Ant Colony, The Louvre

\section{INTRODUCTION}

\subsection{Background}

Each year there are countless lamentable emergency evacuation failures being reported[1]. Especially, for the large areas with thousands of tourists, emergency evacuation plan plays an important role in ensuring the safety of the people inside, as it helps individuals leave the building as quickly and safely as possible. However, some previous studies [2,3] point out that with the lack of gates and complex structure, it would be rather difficult to evacuate all the people within the building.

In general, to help reduce the evacuation time and identify the bottlenecks during evacuation, it's of vital importance to obtain a evacuation simulation model, as it helps the administrators to get a better understanding of the building and also makes it easier for them to work out an evacuation plan.

Natarajan Meghanathan et al. (Eds) : CSEIT, CMLA, NeTCOM, CIoT, SPM, NCS, WiMoNe, Graph-hoc - 2019 pp. 45-56, 2019. (C) CS \& IT-CSCP 2019

DOI: $10.5121 /$ csit.2019.91304 


\subsection{Literature Review}

In order to simulate the flow of people, a series of mathematical models are adopted, such as cellular automaton [4,5], which use the status change of a cellular to represent the movement of an individual. As the individuals in the cellular automaton move without intelligence and insight, while implementing the cellular automaton, some people seek to put the interaction between individuals into consideration and obtain the optimized route using ant colony algorithms $[6,7]$. After that, the cellular automation are modified with spatial refinement [8], which simulates the flow of people better. The cellular automaton is combined with artificial potential field [9] to generate a more precise route for individuals.

Unfortunately, most of these above don't depict the flow of people during the evacuation process precisely, as they haven't fully considered the intelligence of people and the interplay between individuals. Moreover, above research concentrates on evacuation in single simple room [4], which may go uncertain in large building with complex structure. Therefore, though there are many models existing, improvements are still needed.

\subsection{Our Work}

In order to carefully simulate the evacuation process in large complex buildings, a hybrid model integrating 3 sub models is developed, in which human movements at a finer granularity are depicted, and others' influence on the individual is considered.

The remaining part of this paper is arranged in the following order. How to develop this evacuation model from the three aspects of the simulation is given in Section 2. The second floor of the Louvre in France is utilized as an example for the model application in Section 3. Finally, some concluding remarks and the directions of subsequent research are provided in Section 4.

\section{Evacuation Model DevelopMent}

Intuitively, this hybrid model is divided into three sub models, namely Refined Cellular Automaton(RCA), Adapted Artificial Potential Field(AAPF), and Adapted Ant Colony Model(AAC), so that the flow of people during evacuation is simulated more realistically based on those three models.

\subsection{Refined Cellular Automaton}

RCA simulates the movement of individuals inside the building with complex structure. In this model, individuals will move in the direction where there is still place for them to move. As they don't have intelligence, individuals largely move randomly. According to the traditional CA[10], time and space are discretized, and the evacuation area is divided into discrete grids(cells). One cell represents the area taken up by an individual currently. However, such simulation is not fine enough in an evacuation scenario, as the speed of individuals are not carefully depicted, thus it is refined to get the RCA sub model.

\subsubsection{Design of RCA}

In RCA, in order to depict the speed of an individual and portray the evacuation process in more 
detail, the original CA and the modifications are made in the model are listed as follows. The evacuation area are divided into more dense grids, and each grid corresponds to one sub- cell. Each cellular will cover several sub-cells (and in our model a cell occupies a $3 \times 3$ piece of subcells). When an individual moves, its movement will cover several sub-cells. The more sub-cells it covers per unit time, the faster it moves.

When a cell is determining its direction of movement, it will scan for the information contained in its Von Neumann type neighbor sub-cells[11]. After calculation, it will choose a most promising direction.

\subsubsection{Mathematical Model of RCA}

According to the idea above, RCA model is proposed. The individual's velocity is $V$ as shown.

$$
|V(i, j)|=\min \left\{v_{0}, \max \left\{n_{i+1, j}, n_{i-1, j}, n_{i, j+1}, n_{i, j-1}\right\}\right\}
$$

$C(i, j)$ is the cell of row $i$, column $j$. In $(1), V(i, j)$ represents the velocity of the individual in $C(i, j)$. And $v_{0}$ is the velocity of this individual regardless of his surroundings. Then, $n_{a, b}$ is defined as (2).

$$
n_{a, b}=\min \left\{d_{\left|(i, j)-\left(a_{k}-b_{k}\right)\right|}\right\}
$$

In (2), $\left(a_{k}, b_{k}\right)$ denotes all the cells where the individual has an overlap with $C(a, b)$. Moreover, $d_{\left|(i, j)-\left(a_{k}-b_{k}\right)\right|}$ represents the number of sub-cells between $C(i, j)$ and $C\left(a_{k}, b_{k}\right)$.

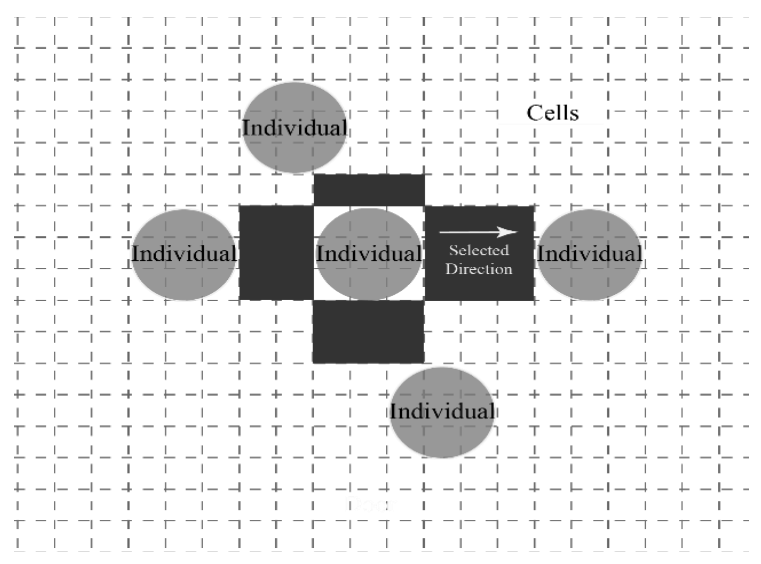

Figure 1: Individual's choice of moving direction

\subsection{Adapted Artificial Potential Field Model}

This sub model enables the individuals in our model to know the path to the doors when they are inside a room.

Artificial Potential Field(APF) serves as a method for local path planning[12,13], by assigning potential energy to the area and generating an APF. The object inside is able to get the route by setting the direction as the descending direction of the potential direction. Thus, it is integrated into this model so as help the individuals to get the route to the door. 
However, APF appears to be weak when it comes to buildings with complex structures. In APF, the potential energy increases in line with the straight-line distance to the source. That means, when obstacles exist between the object and the source, the object cannot find a way to avoid them. Thus, the object in APF easily gets trapped when there are complex obstacles in the scene, or the room is U-shaped.

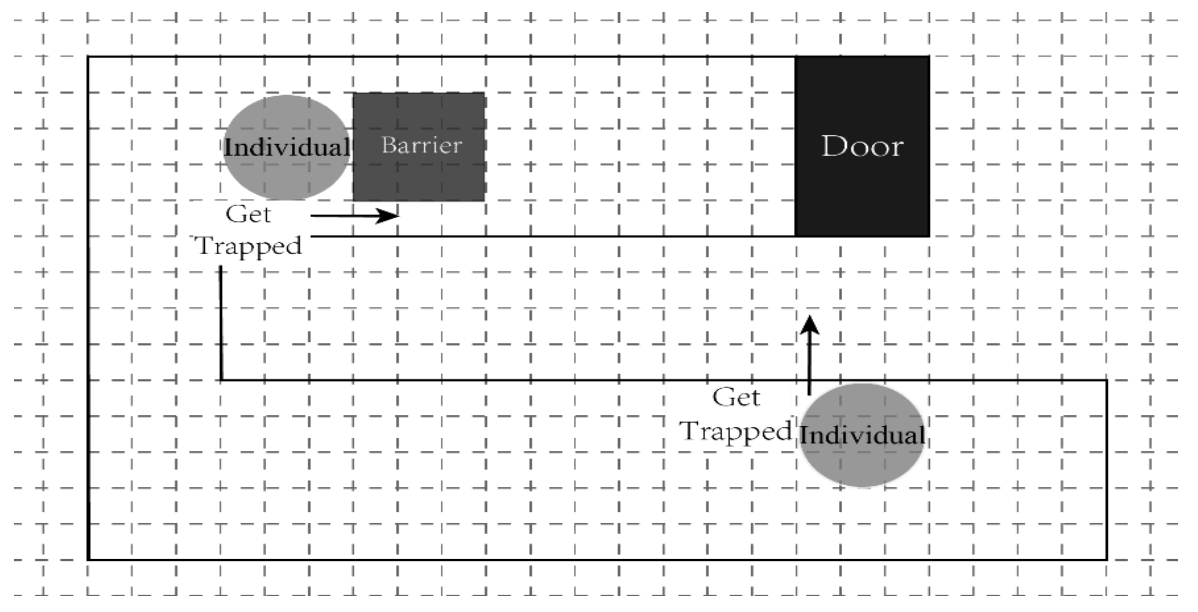

Figure 2: Individual gets trapped in APF under certain scenarios

To solve the problem, APF is modified as Adapted Artificial Potential Field(AAPF) in this hybrid model. The route is simulated along which the visitors will go for the doors spontaneously during evacuation if they are inside a room.

\subsection{Design of AAPF}

In AAPF, the distribution of potential energy within the area is modified so that the individuals inside can get to the door without being trapped by obstacles or the corners of the room.

The potential energy increases along the route in the room, not in line with the straight-line distance to the source. In this way, obstacles and corners inside the room will have no influence on the routes of the individuals as the distribution of potential energy has already bypassed them.

The doors are set as destinations, which possess the lowest potential energy. Thus, individuals inside a room will spontaneously move towards the doors, which is consistent with the reality.

AAPF and RCA are actually closely intertwined in an evacuation scenario. While RCA just depict the movement of the individuals, AAPF provides intelligence for the individuals, and enables them to 'see' the doors when they are inside a room and go for it instead of moving without an aim. Also, the potential energy is left in each cell, that is to say, by reading the potential energy left in his surrounding cells and getting the lowest one, the correct direction to the door is known by individual. 


\subsubsection{Mathematical Model of AAPF}

The potential energy of cells and attraction of doors are normatively denoted as the following two functions. The potential energy of the $i^{\text {th }}$ visitor when one is in the $h^{\text {th }}$ room at the time $t$ is

$$
U_{h t}(i)=12 \cdot \xi \cdot((i))^{2}
$$

with $\xi$ a constant. In (3), $\rho_{g t}(i)$ represents the Euclidean distance between the $i^{\text {th }}$ visitor and the $g^{\text {th }}$ door at time $\mathrm{t}$ where the $g^{\text {th }}$ door means the door that the $i^{\text {th }}$ visitor has selected. After that, the door's attraction is as follows.

$$
F_{h t}(i)=-\nabla[h(i)]=\xi \cdot\left(\rho_{g t}(i)\right)
$$

(4) shows the door's attraction to the $i^{\text {th }}$ visitor at time t with The potential energy of the $i^{\text {th }}$ visitor when one is in the $h^{\text {th }}$ room at the time $t$ in (3).

\subsection{Adapted Ant Colony Model}

The influence of people's own thoughts and minds of the simulation is included in this sub model. That is, individuals will be affected by others' choice when deciding which door they move towards.

The original Ant Colony Algorithm simulates the phenomenon in an ant colony, that the latter tends to follow the former along the pheromone left behind while they are moving. By tracking the pheromone whose concentration is inversely proportional to the length of the path, the ants are able to identify the optimal route[14]. To simulate the herd mentality of the individuals in the process of evacuation, it is integrated into this model so that the evacuation process is simulated more precisely.

In order to make it adapted to the situations where the structure of the building is complex, the phenomenon has to be left on the doors rather than along the route. Thus, the original Ant Colony model is modified to be Adapted Ant Colony model (AAC), so that it simulates how each individual's choice is influenced by others.

\subsubsection{Design of $\mathrm{AAC}$}

In the adapted ant colony model, the modifications to the original Ant Colony Model are listed as follows.

The pheromone is only left on the door rather than alongside the specific path that an individual walk along. Until an individual arrives at one of the exits of the Louvre, the pheromone he spread begin to take effect. The numbers of doors on the route are to indicate the length of the route instead of using the actual path length.

That's to say, AAC simulates the process that every visitor chooses a specific door as his destination when he is inside a room. 


\subsubsection{Mathematical Model of AAC}

In this paper, the probability that each visitor chooses a door is

$$
p_{i j}^{k}(t)=\frac{\left[\tau_{i j}(t)\right]^{\alpha} \cdot\left[\eta_{i j}(t)\right]^{\beta}}{\sum_{s \in D_{k}(t)}\left[\tau_{i s}(t)\right]^{\alpha} \cdot\left[\eta_{i s}(t)\right]^{\beta}} .
$$

In (5), $\tau_{i j}(t)$ and $\eta_{i j}(t)$ respectively represent the concentration of pheromone at the $j^{\text {th }}$ door and the inverse of the distance towards the $j^{\text {th }}$ door at the time of $t$ when the $i^{\text {th }}$ visitor is at the $i^{\text {th }}$ door. $\alpha$ and $\beta$ respectively mean the weight index of $\tau_{i j}(t)$ and $\eta_{i j}(t)$. In addition, $D_{k}(t)$ refers to the doors that are available and passed by some successful visitors for the $k^{\text {th }}$ visitor at time $t$.

As RCA considers the influence of the distance towards the doors inside a room, so weight $\beta$ equals zero. As the concentration of the pheromone increases, the effect of the distance between $D_{i}$ and $D_{j}$ is ignored.(5) gives us the probability of the $k^{t h}$ visitor to choose the $j^{t h}$ door when he is at the $i^{\text {th }}$ door at time $t$.

The way to get $\tau_{i j}(t)$ is as follows.

$$
\tau_{i j}(t+\Delta t)=\rho \cdot \tau_{i j}(t)+\sum_{h \in S_{k}} \Delta \tau_{i j}^{h}
$$

In (6), the time unit is set as $\Delta t$, where $\rho$ represents the probability that pheromone is retained within $\Delta t$. Then $S_{k}(t)$ means those successful visitors that has passed door $_{j}$ throughdoor ${ }_{i}$ during $\Delta t$ after time $t$ with

$$
\Delta \tau_{i j}^{h}=\frac{1}{L_{h}}
$$

$L_{h}$ represents the number of the doors that the successful visitors have passed after door . $\tau_{i j}(t+\Delta t)$ is obtained by calculating $\tau_{i j}(t)$ using methods mentioned in (6).

\section{Model ApPlication ANd Analysis}

Based on the hybrid model developed, it is applied to real-life scenarios to test the applicability. Here our model is applied to the Louvre to simulate an evacuation process with some real data got from Affluences[16]. After building the evacuation simulation, the evacuation process is analyzed and the bottlenecks are identified.

The Louvre has witnessed a series of shocking terror attacks taken place in France since 2012[17], and they have put French citizens as well as tourists at threat. Being one of the largest and the most popular art museum in France, the Louvre accepts an average of 15,000 visitors a day[18]. Thus, the Louvre needs a comprehensive and adaptable evacuation model, so that in the event of an emergency, its internal visitors can evacuate smoothly and minimize losses. That's why the Louvre is chosen to test our hybrid model. 


\subsection{Model Application}

In order to apply the hybrid model to the Louvre precisely, data are gathered including the size[19] and the floor plan of the Louvre[20], and also get the real-time tourists' number from Affluences[12]. Using the data gathered, the 2nd floor model is constructed based on the floor plan. The halls into rooms are also divided according to the serial numbers in the floor plan. Numbers in Fig 3 are serial numbers of rooms.

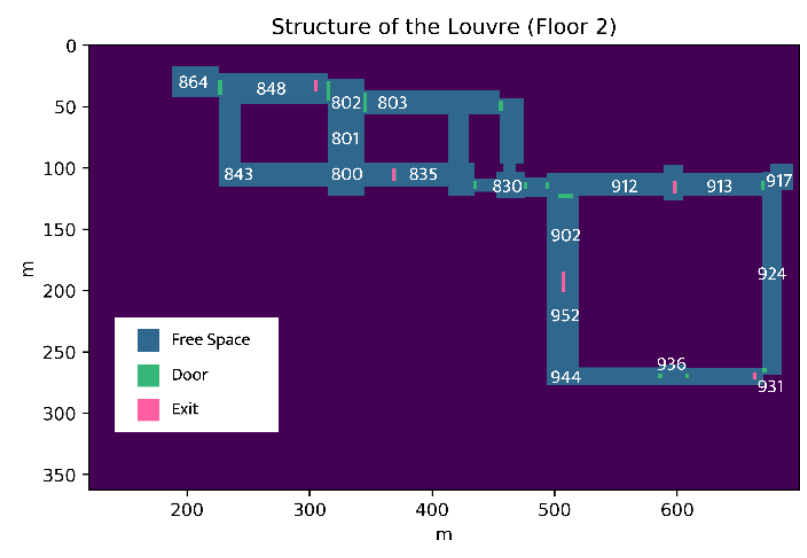

Figure 3: Structure of the Louvre (Floor 2)

\subsubsection{Assumptions and Preparation}

In order to simplify the course of modeling and draw some reasonable conclusions from the model, assumptions are as follows:

1.All the visitors will follow the guidance during evacuation.

2.When visitors arrive at the exits, they are able to leave the Louvre successfully. It is assumed that there won't be congestion outside the exits when emergencies happen.

3.The moving speed of the visitors are divided into two groups, and people within the same group move at a same speed. It's assumed that the visitors are divided into two groups: people who walk at normal speed, people who walk slower. The people who walk slower means those who have difficulty in moving, the elderly and the children for example.

Also, visitors are distributed to the five floors based on the floor area ratio of five floors. In this model, the number of visitors on the $2^{\text {nd }}$ floor is 133.

\subsubsection{Application of the Sub Models}

Three sub models of this hybrid model are applied to the $2^{\text {nd }}$ floor of the Louvre. Here how each of these sub models are applied to the scenario of evacuation is illustrated.

\section{Refined Cell Automaton}

After dividing the area into more dense grids, RCA has the ability to depict the speed difference between different kinds of individuals, such as the elderly and people at a younger age, by letting them cover a different number of cells at the same time. 


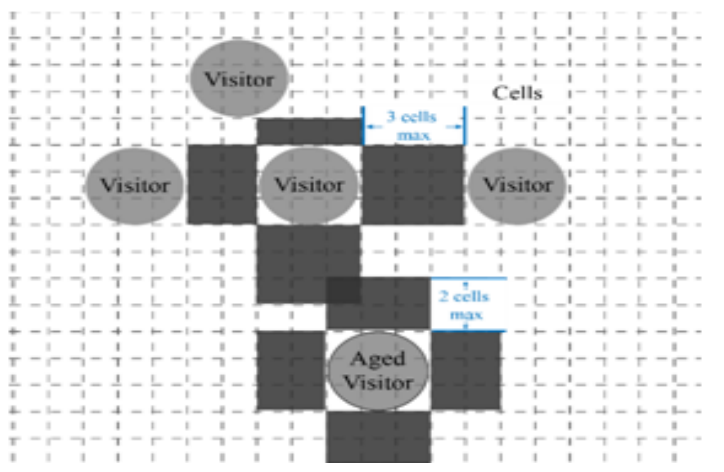

Figure 4: Speed difference between different kinds of visitors described using RCA

\section{Adapted Artificial Potential Field}

The route to the door for the individual inside a room is obtained using by the model of AAPF, as there are potential energy restored in each cells. The direction of an individual in which the cells have the lowest potential energy is chose by reading the potential energy in his surrounding cells and comparing them.

The choice of direction of a visitor in AAPF is shown in Figure 5. The gradient of color depicts the potential energy in the room. The darker the color, the greater the potential energy is here. Thus, the visitors inside will choose to move towards where the potential energy is relatively lower, that is, where the color is relatively lighter.

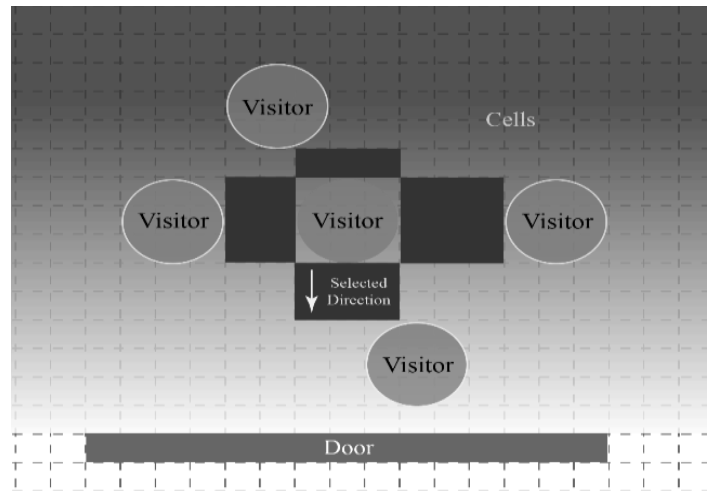

Figure 5: Choice of direction of visitor in AAPF

\section{Adapted Ant Colony Model}

AAC enables individuals to be affected by other individuals. When one individual gets to the exit and leave the building, pheromone will be left on the doors he passed. Combing with AAPF, the door on which the pheromone was left will be able to create an AAPF within the room, enabling the individuals inside to be guided to this door. Through such method, the individuals behind will be able to know through which door they can get to the exit.

Figure 6 shows the pheromone a visitor left after he arrived at the exit successfully. The arrows indicate the marks he left on the doors in his forward route. 


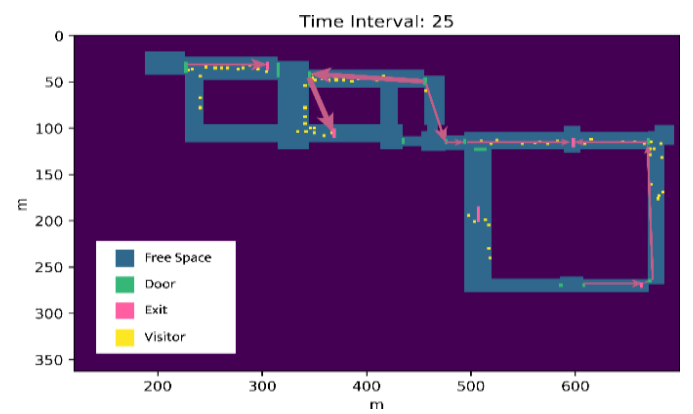

Figure 6: Pheromone a visitor left after he arrived at the exit

\subsection{Analysis of the Simulation Result}

Python is used to construct our simulation program, and matplotlib was utilized to draw the diagrams representing the evacuation situation. Every second, the current evacuation situation is updated in the last second and displayed via matplotlib. Different kinds of visitors are displayed in different kinds of colors, thus they can be easily recognized in the plot.

Through simulation, it is found out that the total evacuation time of the people on the $2^{\text {nd }}$ floor is 91 seconds with 133 people. The bottlenecks in the $2^{\text {nd }}$ floor are also identified, which is specifically located at the exhibition hall 800, 802, 803 and the door between exhibition hall 802 and803. At the same time, in Figure 7,the evacuation situation of the $2^{\text {nd }}$ floor at some specific time points during the evacuation process is shown.

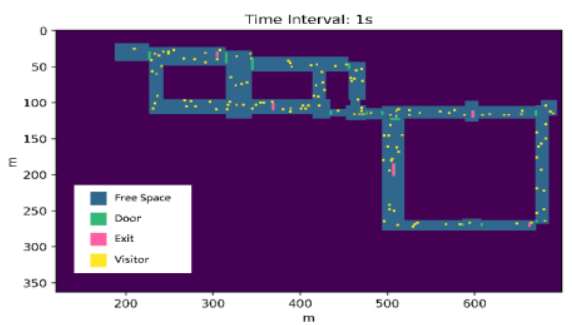

(a)

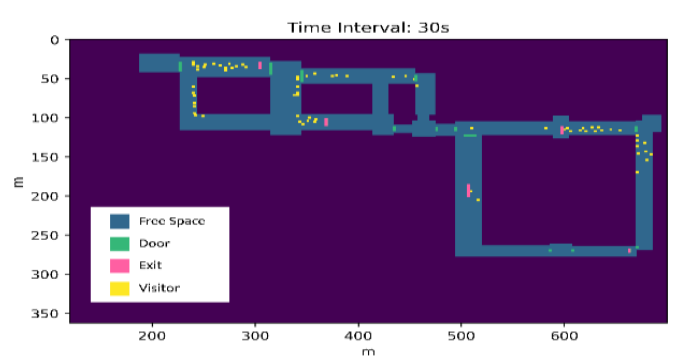

(c)

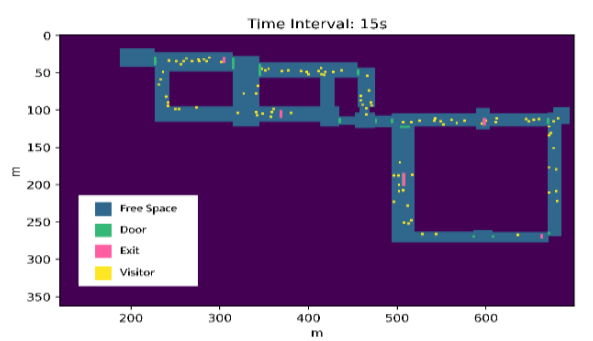

(b)

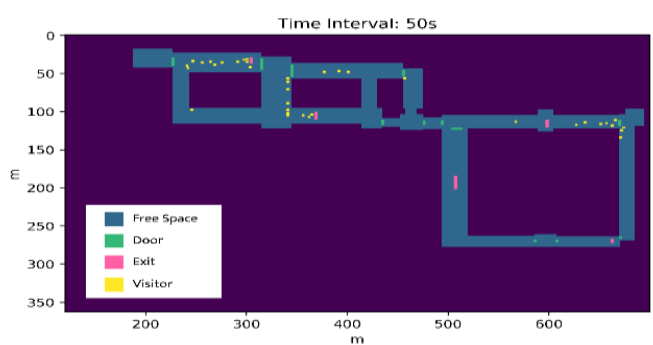

(d)

Figure 7: Evacuation situation of the Louvre at

(a) $1^{s t}$ second (b) $15^{t h}$ second (c) $30^{t h}$ second (d) $50^{t h}$ second

With the evacuation process precisely depicted in Figure 7, the congestion along the evacuation route is easily distinguished with naked eye as shown in Figure 8 . They are specifically located at the exhibition hall 800, 802, 803 and the door between exhibition hall 802 and 803 . 


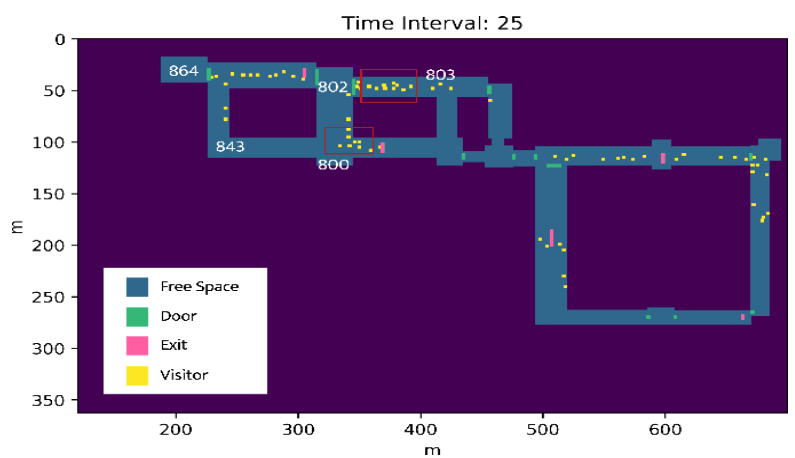

Figure 8: Congestion at 25th second

\section{Conclusions}

Based on CA, the model is refined by dividing the area into more dense grids. Combined it with AAC and AAPF which give the individuals the ability to find their way to the doors and let them be alerted by their predecessors who have already get to the exits, a hybrid model is established. This hybrid model is applied to simulate the evacuation process inside the large buildings with complex structure with great intuition and preciseness.

The hybrid model is applied in the Louvre to simulate the evacuation process with real-time data. The evacuation situation during the whole process is obtained, and the bottlenecks of the evacuation route are identified. During the process, the speed difference of the elderly and those at a younger age is taken into consideration. This has proved the high applicability and flexibility of the hybrid model.

The future work could fruitfully explore this issue further by looking into optimizing the speed of AAPF model, and looking into applying the hybrid model to a multi-story building. Also, some research can be done to analyze the influence of stairs and lifts to the hybrid model, so that the hybrid model can be applied to more complex situations.

\section{REFERENCES}

[1] J. SERNA, P. ST. JOHN and R. LIN II, "Disaster after disaster, California keeps falling short on evacuating people from harm's way", 2019. [Online]. Available: https://www.latimes.com/local/lanow/la-me-paradise-fire-evacuation-system-20181120-story.html. [Accessed: 24- May- 2019].

[2] J. Wang, "Uncertainty study on large-scale crowd evacuation in unconventional emergencies", Doctor, University of Science and Technology of China, 2013. (in Chinese)

[3] X. Zhou, "Analysis of safe evacuation of metro stations", Encyclopaedia Form, vol. 17, p. 263, 2018. [Accessed 24 May 2019]. (in Chinese)

[4] T. Ping, "Research on Personnel Evacuation Simulation Based on Cellular Automata Model", Computer Simulation, vol. 10, no. 26, pp. 319-322, 2009. [Accessed 24 May 2019].(in Chinese)

[5] H. Feng, J. Yang, B. Liu and J. Zhu, "Simulation of pedestrian flow evacuation based on cellular automata", Construction Science and Technology, no. 7, pp. 64-66, 2017. [Accessed 24 May 2019]. (in Chinese) 
[6] Q. Du, R. Chen and A. Xu, "Subway pedestrian evacuation model based on ant colony cellular automaton", Computer era, no. 2, pp. 18-21, 2018. [Accessed 24 May 2019]. (in Chinese)

[7] J. Fu, Y. Liu and J. Li, "Fire Dynamic Evacuation Based on Ant Colony Algorithm", Journal of East China Jiaotong University, vol. 34, no. 3, pp. 118-124, 2017. [Accessed 24 May 2019]. (in Chinese)

[8] N. Ta, "Modelling and simulation of pedestrian evacuation based on spatial refinement cellular automata", Master, Inner Mongolia university, 2016. (in Chinese)

[9] R. He, "Simulation of crowd evacuation based on potential energy field model", Master, Sun Yat- sen University, 2019. (in Chinese)

[10] Z. JIN, X. Ruan and L. Li, "Evacuation Simulation in Narrow Passage Under Fire Scenario Based on Cellular Automaton", Journal of Tongji University(Natural Science), vol. 46, no. 8, pp. 1026-1034, 2018. [Accessed 24 May 2019]. (in Chinese)

[11] C. Langton, "Self-reproduction in cellular automata", Physica D: Nonlinear Phenomena, vol. 10, no. 1-2, pp. 135-144, 1984. Available: 10.1016/0167-2789(84)90256-2.

[12] D. Li, L. Yuan, Y. Hu and X. Zhang, "Large-scale crowd motion simulation based on potential energy field", Journal of Huazhong University of Science and Technology(Nature Science Edition), vol. 44, no. 6, pp. 117-122, 2016. [Accessed 24 May 2019]. (in Chinese)

[13] Z. Wu, "Evacuation simulation of crowds in unfamiliar environments", Master, SunYat-sen University, 2010. (in Chinese)

[14] Y. Yan, "Research and application of ant colony algorithm", China Venture Capital, no. 3, pp. 201 202, 2019. [Accessed 24 May 2019]. (in Chinese)

[15] Q. Wu and L. Wang, Intelligent Ant Colony Algorithm and Application. Shanghai: Shanghai Science and Technology Education Press, 2004. (in Chinese)

[16] "Affluences - L'affluence en temps réel", Affulences, 2019. [Online]. Available: https://www.affluences.com/louvre.php. [Accessed: 24- May- 2019].

[17] T. Reporters, "Terror attacks in France: From Toulouse to the Louvre", The Telegraph, 2018. [Online]. Available: https://www.telegraph.co.uk/news/0/terror-attacks-france-toulouse-louvre/. [Accessed: 24- May- 2019].

[18] "Online Extra: Q\&A with the Louvre's Henri Loyrette", Bloomberg.com, 2002. [Online]. Available: https://www.bloomberg.com/news/articles/2002-06-16/online-extra-q-and-a-with-the- louvres-henriloyrette. [Accessed: 24- May- 2019].

[19] "The "Pyramid" Project (2014-2016)", Louvre.fr, 2014. [Online]. Available: http://www.louvre.fr/sites/default/files/dp_pyramide\%2028102014_en.pdf. [Accessed: 24- May2019].

[20] "Louvre - Interactive Floor Plans | Louvre Museum | Paris", Louvre.fr, 2019. [Online]. Available: https://www.louvre.fr/en/plan. [Accessed: 24- May- 2019]. 


\section{AuTHORS}

Corresponding author:

Xiongda Chen, professor, majored in operational research and cybernetics.

First Author Equally:

Hao Yuan, undergraduate, majored in the study and research of digital media.

Guo $\mathbf{Y u}$, undergraduate, majored in the study and research of mathematical statistics.

Yifan Ma, undergraduate, majored in the study and research of software management.

\section{Second Author:}

Jieneng Chen, undergraduate, majored in the study and research of computer science 